\title{
Chamber catalogues of optical and fluorescent signatures distinguish bioaerosol classes
}

\author{
Mark Hernandez ${ }^{1}$, Anne E. Perring ${ }^{2,3}$, Kevin McCabe ${ }^{4}$, Greg Kok $^{5}$, Gary Granger $^{5}$, and Darrel Baumgardner ${ }^{5}$ \\ ${ }^{1}$ Department of Civil, Environmental and Architectural Engineering, UCB 428, University of Colorado, \\ Boulder, CO 80309, USA \\ ${ }^{2}$ National Oceanic and Atmospheric Administration, 325 Broadway, Boulder, CO 80305, USA \\ ${ }^{3}$ Cooperative Institute for Research in Environmental Sciences, University of Colorado, Boulder, CO 80309, USA \\ ${ }^{4}$ Department of Sciences, Columbia George Community College, 400 East Scenic Drive, The Dalles, OR 97058, USA \\ ${ }^{5}$ Droplet Measurement Technologies, 2545 Central Ave, Boulder, CO 80301, USA \\ Correspondence to: M. Hernandez (mark.hernandez@colorado.edu)
}

Received: 2 December 2015 - Published in Atmos. Meas. Tech. Discuss.: 19 January 2016

Revised: 29 June 2016 - Accepted: 29 June 2016 - Published: 26 July 2016

\begin{abstract}
Rapid bioaerosol characterization has immediate applications in the military, environmental and public health sectors. Recent technological advances have facilitated single-particle detection of fluorescent aerosol in near real time; this leverages controlled ultraviolet exposures with single or multiple wavelengths, followed by the characterization of associated fluorescence. This type of ultraviolet induced fluorescence has been used to detect airborne microorganisms and their fragments in laboratory studies, and it has been extended to field studies that implicate bioaerosol to compose a substantial fraction of supermicron atmospheric particles. To enhance the information yield that newgeneration fluorescence instruments can provide, we report the compilation of a referential aerobiological catalogue including more than 50 pure cultures of common airborne bacteria, fungi and pollens, recovered at water activity equilibrium in a mesoscale chamber $\left(1 \mathrm{~m}^{3}\right)$. This catalogue juxtaposes intrinsic optical properties and select bandwidths of fluorescence emissions, which manifest to clearly distinguish between major classes of airborne microbes and pollens.
\end{abstract}

\section{Introduction}

Fluorescence aerosol interrogation is gaining increased attention for its ability to characterize particulate matter suspended in both indoor and outdoor environments (Huffman et al., 2010; Sivaprakasam et al., 2011). When simultane- ously reporting from multiple bandwidths, ultraviolet induced fluorescence (UV-LIF) has successfully detected airborne microbes in bench-scale chamber studies (Healy et al., 2012; Kaye et al., 2005; Toprak and Schnaiter, 2013) and been applied to support large-scale aerosol monitoring campaigns where fluorescent particle cohorts indicated significant bioaerosol contributions to airborne organic carbon pools (Gabey et al., 2010; Pöschl et al., 2010; Gabey et al., 2013; Perring et al., 2015).

A variety of biogenic fluorophores have evolved in microorganisms and pollen grains, many of which are relevant to UV-LIF characterization of aerosols (Lakowicz, 2006; Poehlker et al., 2012). These fluorophores include metabolic mediators (e.g., nicotinamide adenine dinucleotide hydrogenase (NADH) and its phosphorylated derivatives, NADPH) that are widely conserved throughout the microbial and plant worlds. Other fluorescently active biopolymers include a wide variety of structural proteins and pigments; however, these microbial compounds are tremendously variable in conformation and intracellular quantity (Kepner and Pratt, 1994; Madigan et al., 2008; Hill et al., 2014). As such, the distribution of fluorescence yields from some (whole-cell) microbial bioaerosols has been reported to have sensitivities to age (Kanaani et al., 2007) as well as cultivation history and environmental conditions (Saari et al., 2014; Uk Lee et al., 2010).

Sensitivity analyses of bioaerosol spectra from different UV-LIF instrument configurations are emerging in the litera- 
ture (Agranovski et al., 2003; Huffman et al., 2010; Poehlker et al., 2012), as are generalized performance indices designated as apparatus-specific detection efficiencies (Saari et al., 2014). These UV-LIF metrics include, but are not limited to, photomultiplier thresholds, fluorescent particle fraction (FPF) recoveries and specific quantum yield comparisons. The calibration of modern UV-LIF instruments has been predominantly executed with thermoplastic spheres that are impregnated with colloidal metals or artificial fluorophores. While conventional microsphere calibrants are well characterized from a chemical perspective, they are poor biophysical analogues to the bioaerosols they are meant to reference, and they have sensitive stability windows limited to the order of weeks in cold, dark storage. In this context, no pure culture(s) of microbial bioaerosol, under any formally defined suite of cultivation and aerosol challenge conditions, has yet to be broadly recognized as a UV-LIF calibrant.

There is increasing interest in the utility of UV-LIF for environmental and autecological studies (Despres et al., 2012), yet there remains no unified approach for calibrating and cataloguing optical properties of fluorescence emissions associated with primary biological airborne particles (PBAPs). While many PBAPs fluoresce, interpreting UV-LIF measurements for bioaerosol characterization presents significant interpretive challenges, some of which have been successfully addressed using cluster analyses (Pinnick et al., 2013; Gabey et al., 2013; Crawford et al., 2015; Pan et al., 2015). In addition to classic Bayesian clustering approaches, referential methods can also be applied to analyze fluorescence distributions with selected optical properties, which may in turn be leveraged for environmental comparison(s). To this end, limited laboratory characterizations of some common bioaerosols have been introduced to different UV-LIF instruments, including single- and multiple-wavelength whole-cell microbial challenges in a variety of equipment configurations (Sivaprakasam et al., 2011; Healy et al., 2012; Saari et al., 2014). Some recent UV-LIF studies have demonstrated a library-based approach and specifically cite a need for expanding the database of intrinsic fluorescence properties associated with different PBAPs (Healy et al., 2012). In response, a unified approach for cataloguing intrinsic fluorescence from pure cultures of airborne bacteria (14), fungal spores (29) and pollen grain isolates (12) using a Wideband Integrated Bioaerosol Sensor (WIBS; Droplet Measurement Technologies, Boulder, CO, USA) is presented here. Using a mesoscale $\left(1 \mathrm{~m}^{3}\right)$ bioaerosol chamber, a methodical compilation of optical and fluorescence properties is reported at a defined humidity range, residence time and cell cycle. This serves as a referential model that can be reproduced, expanded and/or modified for economically cataloguing intrinsic bioaerosol fluorescence observations from UV-LIF instruments, under carefully controlled environmental conditions.

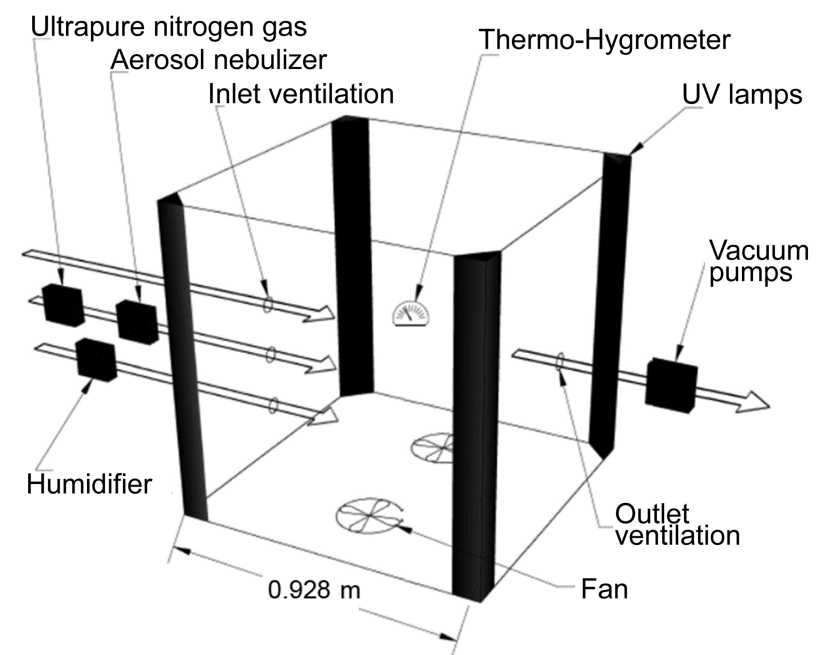

Figure 1. Schematic of bioaerosol test chamber. In independent trials, dry ultrapure nitrogen aerosolized and diluted different pure cultures of bacteria, fungi and pollens into a temperature $\left(20^{\circ} \mathrm{C}\right)$ and RH (25-40\%)-controlled chamber (890 L). Bioaerosol delivery vessels were fitted with steel ports, and all gas streams were contained in $0.4 \mathrm{~cm}$ conductive neoprene tubing, carrying $151 \mathrm{kPa}$ at a flow rate of $12 \mathrm{~L} \mathrm{~min}^{-1}$ into the chamber.

\section{Materials \& methods}

A cohort of light-scattering and fluorescent bioaerosol spectra were obtained from consecutively aerosolizing pure cultures of bacteria, fungi and pollens in an environmentally controlled chamber. The chamber into which all bioaerosol cultures were introduced is cubic $\left(\mathrm{ca} .1 \mathrm{~m}^{3}\right.$ ) and constructed of grounded, $1.25 \mathrm{~cm}$ (thick) Lucite; chamber air was continuously mixed by two $1.5 \mathrm{~W}$ fans (Fig. 1). Comprehensive details of chamber construction and its operation for pure bioaerosol culture characterization have been previously described (Peccia et al., 2000). The WIBS used in this study was placed immediately adjacent to the outside chamber and continuously drew bioaerosol from the chamber interior at $1.2 \mathrm{~L} \mathrm{~min}^{-1}$ through $0.25 \mathrm{~m}$ of conductive neoprene tubing for these tests. The chamber was vented to a hood at ambient pressure (ca. $88 \mathrm{kPa}$ ); relative humidity $(\mathrm{RH})$ was held between 25 and $40 \%$; temperature was held between 20 and $22^{\circ} \mathrm{C}$; and bioaerosol was allowed to equilibrate to these conditions for more than $5 \mathrm{~min}$ prior to UV-LIF sampling.

\subsection{Bacteria}

Fifteen pure bacterial cultures were grown into early stationary phase using standard protocols described by the American Type Culture Collection (ATCC) and German Collection of Microorganisms and Cell Cultures (DSMZ). These cultures, listed with their accompanying names (Table A1 in Appendix A) and fluorescence distributions (Fig. 2), have been used as models for the environmental behavior of airborne bacteria (Hurst et al., 2007). As judged by tracking the 

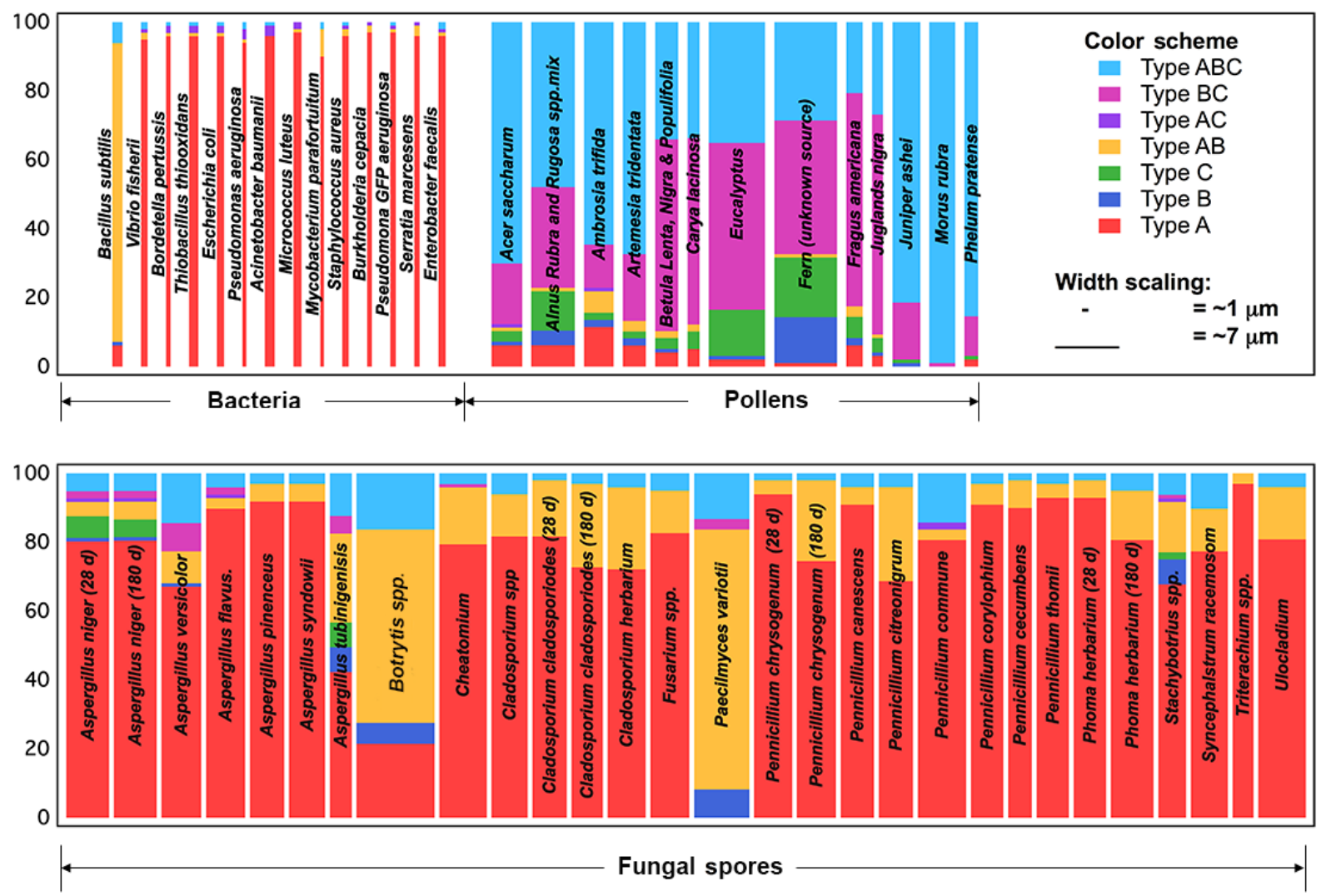

Figure 2. Juxtaposition of optical and fluorescence properties of aerosolized pure cultures of bacteria and pollens (top), and fungal spores (bottom). Fluorescence type distribution is defined by excitation and emission from any of three possible channels alone (A, B or C) or in any combination. Bar width is proportional to equivalent optical diameter (scaling shown below color scheme legend in top panel).

optical density of the cultures (Madigan et al., 2008) upon entering stationary phase, bacteria were washed by sequential centrifugations in cold, sterile deionized water and immediately aerosolized into a chamber using a six-jet Collison nebulizer. Direct phase contrast and fluorescence microscopy (Hernandez et al., 1999) showed these bacteria remained intact after inspecting nebulizer reflux immediately following their aerosolization. Based on previous studies with many of the same bacterial models used here, some microbes, particularly Gram negative bacteria harvested in their log growth phase, can experience significant viability losses after being refluxed in Collison nebulizers even after $10 \mathrm{~min}$ (Stone and Johnson, 2002; Thomas et al., 2011). To minimize aerosolization stress and viability variance, bacterial cultures were harvested in early stationary phase, and nebulization times were limited to $2 \mathrm{~min}$.

\subsection{Fungi}

Twenty-nine pure cultures of commonly occurring indoor fungi (Vesper et al., 2007) were cultured until they presented an obvious spore-bearing physiology, after being inoculated on malt extract agar (MEA) and held at $20^{\circ} \mathrm{C}$ and $25 \% \mathrm{RH}$. As judged by direct phase contrast microscopy, copious fun- gal spores were obviously presented by these cultures between 18 and 28 days following their inoculation, depending on the species. These fungal spores, also presented in Fig. 2, were aerosolized directly off their host agar into the chamber using dry ultrapure nitrogen guided through sterile glass containments specifically designed for this purpose (modified fungal spore source strength tester, FSSST; Sivasubramani et al., 2004). Direct microscopy showed these fungal spores were intact and dominated the airborne biomass, with less than $1 \%$ of supermicron bioaerosol particle volume as associated hyphae.

\subsection{Pollen}

Thirteen pure stocks of common temperate tree and grass pollens, collected under conditions similar to those reported by O'Connor et al. (2013), were obtained from a collection maintained at the Denver Botanic Garden. These pollen stocks, also listed in Fig. 2, were aerosolized into the chamber with the same FSSST configuration used for fungi, with notable fractionation of some grains as judged by direct phase contrast microscopy using accepted methods (Taylor et al., 2004). 
To refine and expand upon previous UV-LIF PBAP cataloguing efforts, we chose cohorts of pure cultures of bacteria, fungi and pollens that have been (repeatedly) recovered from aerosols in both indoor and outdoor environments as judged by culturing and microscopy. Of the 14 bacterial cultures used here, all but two are medically relevant to public health and/or bioterrorism (CDC, 2013), and eight cultures have (commonly) been used as bioaerosol models to their persistence under different atmospheric/disinfection conditions (e.g., UV; Peccia and Hernandez, 2006). The three bacterial cultures used here that have not been previously recovered from the atmospheric environment or otherwise used in prior bioaerosol studies (Thiobacillus sp., Vibrio sp. and Enterobacter sp.), were chosen to broaden the range of bacterial phenotypes used for fluorescence challenges such that all major bacterial physiologies were represented: Gram positive, Gram negative, bacterial spores, cocci, bacilli and vibrio (filamentous bacteria were purposely not included). With respect to the fungi and pollens chosen, the same logic applied in choosing which cultures to use for this demonstrative library (challenge): all members of the 26 fungal cultures used and all 13 pollen grain stocks have been commonly recovered from different atmospheric environments (Hurst et al., 2007; Vesper et al, 2007; O'Connor et al., 2013). While certainly not an exhaustive environmental inventory, these cultures cover a broad range of microbial physiologies commonly recovered from the atmospheric environment.

\subsection{Optical spectra acquisition}

The WIBS ( $\mathrm{v}$ 4.0), previously described by Healy et al. (2012) uses dual-wavelength excitation and fluorescence detection, while simultaneously measuring characteristic dimensions from scattered light. The WIBS variant used here (version VI) detects fluorescence of airborne particles by sequential exposure to UV irradiation from a flashlamp filtered at 280 and $370 \mathrm{~nm}$. Fluorescence emitted due to $280 \mathrm{~nm}$ excitation was detected in two wavebands, $310-400$ and $420-650 \mathrm{~nm}$, using dedicated photomultipliers. Fluorescence emitted due to $370 \mathrm{~nm}$ excitation was detected between 420 and $650 \mathrm{~nm}$. Optical diameter was determined by light scattered from exposure to a $635 \mathrm{~nm}$ laser; it is reported here as an equivalent optical diameter (EOD), defined as the diameter of a spherical particle, with a fixed refractive index (relative to that calibrated with 2.0 and $2.8 \mu \mathrm{m}$ latex beads in air between 25 and $45 \% \mathrm{RH}$ ), scattering the same light intensity as the measured bioaerosol. These optical properties were recovered from a minimum of between $10^{3}$ and $10^{5}$ cells, fungal spores or pollen grains. Each pure culture aerosolized was held in the chamber long enough to be at equilibrium with the contained humidity condition ( $>5 \mathrm{~min}$; Peccia et al., 2001), and bioaerosol was collected over at least a $5 \mathrm{~min}$ period, when operating in the following gain modes: fungi and pollens, low gain; bacteria, higher gain. Between each aerosolization challenge, the chamber was evacuated using a high-volume HEPA filter such that total particle counts were below $10^{2} \mathrm{~m}^{-3}$, and the chamber was subsequently purged with ethanol vapor and/or ozone while illuminated with UV light.

Following the annotation introduced by Perring et al. (2015), particle fluorescence was categorized as one of seven types, which considers each of three fluorescence bandwidths individually, as well as in all possible combinations. Here the subscripts denote excitation wavelengths, and parentheses indicate the emission bandwidths observed (nanometers):

$$
\begin{aligned}
& \text { TYPE } A_{280}=(310-400) ; \quad \text { TYPE } B_{280}=(420-650) ; \\
& \text { TYPE } C_{370}=(420-650) .
\end{aligned}
$$

While this notation departs from the fluorescence emission notations used in studies previously describing WIBS performance, it provides a convenient symbolization system for visualizing and discussing fluorescence as described by distributions of emissions. Leveraging these measured quantities, the following metrics were analyzed and compiled for each bioaerosol: (i) the frequency of particles that could be segregated by fluorescence signal (bandwidth) into any of the seven types; (ii) the average fluorescence intensity within each bandwidth (if any); and (iii) the average EOD of each particle type. As is customary with interpreting UV-LIF data (Healy et al., 2012), we report results where a mean forced trigger value plus 3 standard deviations was used as the minimum data recovery threshold.

To demonstrate detection of the potential effects aging can have on intrinsic fluorescence emissions under these conditions, airborne spores of Phoma spp., Penicillium chrysogenum, Cladosporium cladosporioides, and Aspergillus versicolor that were less than 28 days old were compared to the spectra of spores from these same cultures, which were allowed to age 180 days (raised and maintained under identical environmental conditions).

\section{Results \& discussion}

\subsection{Composite optical recognition patterns of bioaerosol classes}

The seven fluorescence categories, the fluorescence intensity and the relative size expressed by the EOD provide a multidimensional matrix from which composite signatures can be constructed for the three bioaerosol classes tested here. Figure 2 shows the EOD and the distribution of fluorescence type for each of the cultures studied, while Fig. 3 shows the normalized fluorescence intensity emitted and composite weighting of their distributions considering all fluorescence types. As judged by EOD and fluorescence type distributions, the pure bioaerosol cultures used to challenge this WIBS distinctly assembled into their major respective physiologic groups. All of the bacterial cultures aerosolized as 


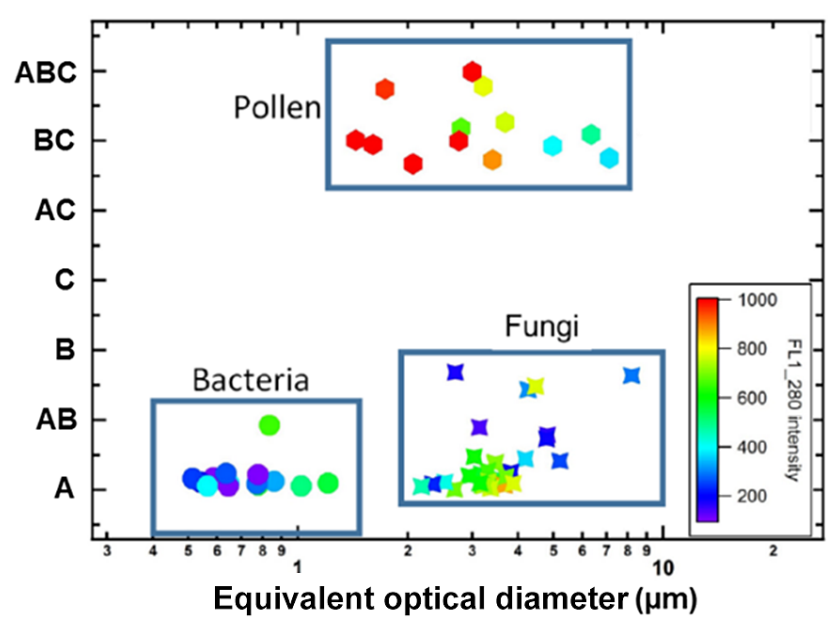

Figure 3. Assemblages of equivalent optical diameter and fluorescence type from aerosolized pure cultures of bacteria $(\bullet)$, fungi spores $(\mathbf{X})$ and pollen grains $(\checkmark)$. The fluorescence type reported is a weighted composite of fluorescent type occurrence observed from each culture or pollen stock aerosolized; intensity is a relative scale.

vegetative cells assembled with common fluorescence patterns below an EOD of $1.5 \mu \mathrm{m}$ and presented the weakest fluorescence intensities of the bioaerosols observed. With the exception of spore-forming Bacillus subtilis, bacterial bioaerosol was dominated by a single fluorescence type (A). The fungal spores aerosolized were in a markedly higher range with respect to their equivalent optical diameters $(2-$ $9 \mu \mathrm{m})$ but presented several fluorescence types: $\mathrm{A}, \mathrm{AB}, \mathrm{BC}$ and $\mathrm{ABC}$, with a prevalence of $\mathrm{A}$ and $\mathrm{AB}$. While the pollens aerosolized were overlapping in equivalent optical diameter ranges with some of the fungal spores observed, they presented significantly different fluorescence type distributions, which were dominated by combinations of fluorescent types $\mathrm{BC}$ and $\mathrm{ABC}$. Note that many of the pollen EODs presented here are considerably smaller than the true diameter of their nascent intact-grain size, which is attributed here to pollen fragmentation during collection, storage or aerosolization. Because of this phenomenon, the EOD distributions for pollen grains are much wider than their fungal or bacterial counterparts, and the means of these distributions are less than unadulterated pollen grains, some of which have true optical diameters larger than $10 \mu \mathrm{m}$. These results are consistent with laboratory and environmental studies which indicate that pollen grain fragmentation routinely occurs in the atmosphere (D'Amato et al., 2007; Miguel et al., 2006; Taylor et al., 2002, 2007). The pollens assembled together based on the ascendancy of type $\mathrm{C}$ fluorescence and their markedly higher fluorescence intensities relative to the other bioaerosol classes observed (Fig. 3).

As judged by WIBS, the multi-channel fluorescence response observed from the seven Penicillium species aerosolized here are in general agreement with those of the Penicillium notatum by Toprak and Schnaiter (2013); they reported fluorescence emissions from P. notatum commensurate with those observed here (dominance of type A, with ascendance of type $\mathrm{C}$ ), where these aerosolized microbes were mixed with ammonium sulfate aerosol in smallchamber studies. Pan et al. (2010) also reported fluorescence (type B) associated with aerosolized B. subtilis spores similar to that reported here. The spectral distributions of $B$. subtilis spores reported here, and by Pan et al. (2010), suggest that these aerosolized spores had markedly different fluorescence than that observed from vegetative bacterial cells with nearly the same EOD. Although they did not aerosolize into bioaerosol chambers which facilitate water activity equilibrium, Pan et al. (2011) also reported a limited catalogue of dual-wavelength fluorescence spectrometer (DWFS) observations by directly introducing 12 dry pollen stocks and four different types of fungal spores to the optical chamber of a DWFS. Of those pollens that overlapped with the genera of the pollen grains used here (Betula spp., Ambrosia spp.) and of those fungal cultures which were the same as those reported here (Aspergillus flavus, Cladosporium herbarum and Penicillium chrysogenum), there were general similarities in fluorescence distributions such that these fungal spore expressed more dominance of type A fluorescence, while the pollens expressed ascendance of type $\mathrm{C}$ fluorescence.

In a cataloguing approach similar to that reported here, Healy et al. (2012) challenged a WIBS 4 with seven different types of fungal spores and eight different pollen grain stocks in a bench-scale chamber under mid-range humidity conditions (ca. 54\%). As was the case here, the pollens aerosolized by Healy et al. (2012) presented a much broader diversity of fluorescence emissions recovered across the three fluorescent WIBS channels when compared with the two Cladosporium spp. they aerosolized; however, the same fluorescence emission pattern was not true when juxtaposing fluorescence observations with that of the other airborne fungi they tested. When comparing fluorescence response of the seven Aspergillus and eight Penicillium species tested here to the response of the single Aspergillus (A. fumigatus) and Penicillium (P. notatum) species reported by Healy et al. (2012), there were significant differences in fluorescence, particularly with respect to the prevalence of type $\mathrm{C}$ fluorescence. Because chamber volume and bioaerosol residence time were undisclosed, it was unclear if the bioaerosol challenges executed by Healy and coworkers were at water activity equilibrium with the chamber humidity. This physical condition, the aerosolization scheme, chamber operations and the limited number of fungi cultures observed may account for these differences. Indeed, Healy and coworkers cited the need for "further studies to generate and add to the current library/database of WIBS-4 particle signatures"; in part, this study responds to that prescribed need and refines culturing, aerosolization and (larger) environmental chamber conditions, and, in particular, equilibrium water activity with respect to the bioaerosol observed. 


\subsection{Fluorescence response to spore aging}

Fluorescence spectra from four pairs of young and aged fungal spores were compared through these analyses (aged 28 vs. 180 days). Of these, two presented no discernable change in the EOD and fluorescence distributions used as cataloguing metrics here: Cladosporium cladosporioides and Aspergillus niger. There was, however, a significant shift in the fluorescence distribution, from younger to older spores, of the species Penicillium chrysogenum and Phoma herbarium (Fig. 2 bottom). More than $90 \%$ of the spores of these species presented their fluorescence as type A when aerosolized at an age of 28 days, but this decreased to near $70 \%$ after 180 days of culture aging under identical conditions, with the balance becoming type AB.

\subsection{Instrumental variability and gain considerations}

We present here the response of a single WIBS instrument to pure cultures of bioaerosols grown, collected and aerosolized under carefully controlled conditions. The instrument manufacturer (Droplet Measurement Technologies) typically sets detector gains based on photomultiplier fluorescence detection from monodisperse challenges with commercially available fluorescent microspheres (polystyrene latex, PSL), the intensity of which is known to vary between manufacturing batches. The fluorescence of PSLs also significantly degrades on the timescale of weeks to months (even with proper cold storage). While this strategy provides general consistency across different instruments, the lack of an absolute calibration means that there can be appreciable variability in fluorescence signal recovery of a particular bioaerosol between one UV-LIF instrument and another. In response, we aerosolized a subset of test bioaerosol cultures to challenge two WIBS in parallel: one owned by the manufacturer, which was used for the larger set of measurements presented here, and another owned by the Chemical Sciences Division of the National Oceanic and Atmospheric Sciences Administration (NOAA). We find differences in spectral classification (Fig. 4), likely attributable to differing gain settings, with the NOAA WIBS recording a larger fraction of a given particle population as having signal above threshold in the $\mathrm{B}$ and $\mathrm{C}$ channels (both of which are detected using the same photomultiplier) than does the manufacturer's market issue WIBS. This operational example highlights a condition that different WIBS instrumentation, while configured the same and challenged under the same conditions, can report somewhat different fluorescence distributions.

\subsection{Implications for real-time detection of atmospheric bioaerosol}

Given the potential variability introduced with culture age and between instruments, genera- or species-type classifications for different bioaerosols should not be treated as ab-
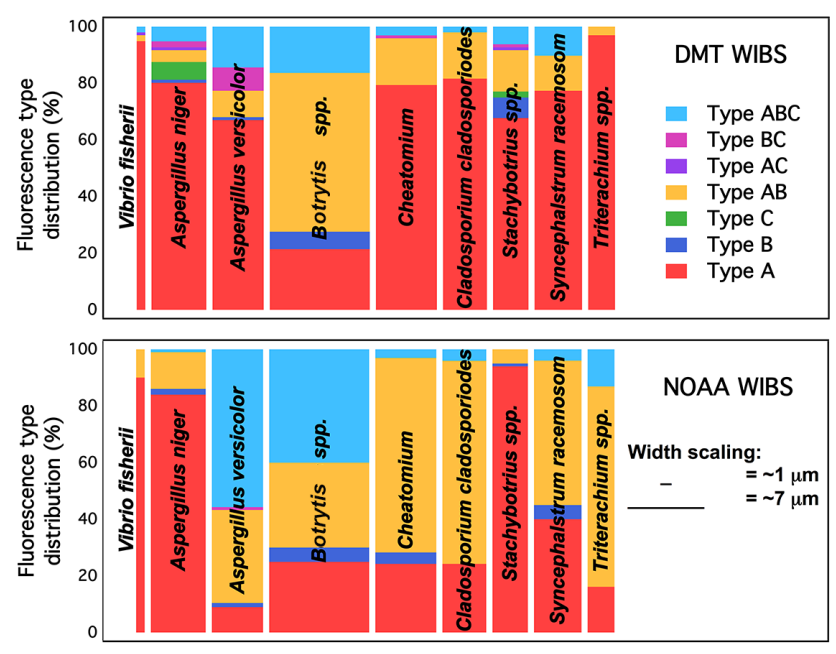

Figure 4. Comparison of fluorescence type distributions for two different WIBS instruments from a subset of the pure bioaerosol cultures and pollen grains used in this analysis. The top panel shows distributions from the DMT WIBS, which was used for all of the bioaerosol chamber challenges presented here. The bottom panel shows the fluorescence type distributions from the NOAA WIBS, used in parallel for a subset of the bioaerosol chamber challenges using pure cultures of bacterial cells and fungal spores.

solute "signatures" but, rather, as likely assemblages that, when considered in conjunction with EOD, allow for discrimination between broad bioaerosol categories. There is also particle-to-particle variability in the type manifestation of a given sample (i.e., not all particles of a given species present the same spectral type) that makes reliable identification on a single-particle basis unlikely and requires, instead, a statistical treatment. For example, bacterial populations tend to present as mostly type $\mathrm{A}$ at small $(<1 \mu \mathrm{m})$ EOD. Fungi present a mixture of type A, AB and ABC fluorescence at larger EOD sizes $(2-9 \mu \mathrm{m})$, although the distribution between the dominant types may differ substantially from that presented here, depending on instrumental parameters, culture circumstances and environmental conditions (e.g $\mathrm{RH}, T$ ). Finally, pollen tends to present a mixture of $\mathrm{C}, \mathrm{BC}$ and $\mathrm{ABC}$ and is the only class of bioaerosol for which types $\mathrm{C}$ or $\mathrm{BC}$ are significant contributors. This facilitates discrimination between fungi and pollen despite the fact that the likelihood of pollen fragmentation reduces confidence in the utility of EOD as a discriminating parameter. Another distinguishing feature of pollens is their relatively high fluorescence intensity, although we note that very little is known about the temporal evolution of fluorescent intensity over atmospherically relevant timescales of photochemical processing.

Type A was dominant in all bacterial and most fungal populations aerosolized in this study (at least for certain gain settings, like those in the DMT WIBS), and thus EOD is an important consideration in distinguishing bacteria from fungi. 
Under laboratory conditions constrained by this work, we typically sampled single, intact, bacterial cells. Atmospheric observations of bacteria and DNA in aerosol, however, indicate that bacteria are frequently found associated with particle sizes larger than that of a single cell. (Burrows et al., 2009; Shaffer and Lighthart, 1997; Tong and Lighthart, 2000; Wang et al., 2008). It is hypothesized that this is due to the presence of either multicell bacterial agglomerates or particle mixtures including bacteria and dust or other vegetative detritus (Bovallius et al., 1978; Lighthart, 1997), and we acknowledge that such agglomerates could be easily confused with intact fungal spores given their spectral similarities as detected by the WIBS. Another notable observation of the ensemble of experiments presented here is the lack of an appreciable fraction of type $B$ particles associated with these pure bioaerosol culture challenges. Type B particles are detected as a minor fraction (up to $15 \%$ ) in only a few fungal and pollen species; therefore, any atmospheric sampling in which type B is a dominant fluorescent type should be examined carefully for potential (non-biological) interferents. Type AC was also strikingly absent from the measurements presented here.

\section{Conclusions}

Recent advances in UV-LIF instrumentation have led to a new generation of atmospheric particle sensors with promising utility for rapid characterization of fluorescent bioaerosols, both indoors and out. Here we have demonstrated the ability of this technique to characterize whole, airborne microbial cells and pollen grains in a relational capacity by refining a chamber-based library approach with environmental conditions controlled at a mesoscale.

Where conventional light-scattering analyses are coupled with the spectral analyses of fluorescence emission(s), a powerful combination of phenotypic information becomes available on a high-throughput, single-particle basis: the broader the excitation and emission spectra observed, the higher the potential for the fundamental characterization of (bio)aerosol in situ. As has been previously described, the WIBS technology employed here demonstrates the potential of multi-channel fluorescence analyses in a laboratory setting; however, this work has refined the approach previously reported by Healy et al. (2012) for expanding existing fluorescence emission catalogues through a simple, unified reference method created under defined chamber conditions. Such a library can be reproduced, expanded and shared by users for interlaboratory comparisons and/or associations with field observations.
In the course of this analysis, the composite evaluation of optical diameter and fluorescent spectra associated with airborne fungal spores, pollens and bacteria revealed that these primary physiologies can be unambiguously differentiated from each other when airborne under defined conditions (culture condition, $\mathrm{RH}$ and temperature). While deeper cluster analyses were beyond the scope of this demonstrative report, within the fungi and pollens there emerged optically defined phenotypes, which also formed distinct subassemblages - an exciting cataloguing outcome with implications for future algorithmic developments and the potential for confident identification of "interferences" which may complicate bioaerosol quantitation in the field (Despres et al., 2012; O'Connor et al., 2013; Poehlker et al., 2012).

The identity of fluorescent aerosol and the fraction thereof composed of primary biological materials remain unknown in many different environments (Despres et al., 2012; Heald and Spracklen, 2009). To this end, these findings suggest that a composite analysis of wideband UV-LIF, as referenced to robust bioaerosol libraries, offers a promising approach to help better characterize airborne particulate matter in the laboratory as well as during wide-area environmental surveillance.

\section{Data availability}

All data (fluorescence counts) recorded, used and analyzed in this investigation are presented in Table A1. Raw WIBS data files can be obtained by request from Droplet Measurements Technologies (darrel.baumgardner@gmail.com). 


\section{Appendix A}

A table containing discrete EOD and intrinsic fluorescence values observed for each culture aerosolized (used to construct Figs. 2 and 3) is presented here.

Table A1. Summary of properties observed for airborne bacteria, fungi and pollens.

\begin{tabular}{|c|c|c|c|c|c|c|c|c|c|c|c|}
\hline \multirow{2}{*}{ Genus species ATCC or DSMZ no. } & \multirow[t]{2}{*}{$n$} & \multirow[t]{2}{*}{ EOD } & \multirow[t]{2}{*}{$\sigma$} & \multirow[t]{2}{*}{ Intensity } & \multicolumn{7}{|c|}{ Fluorescence type frequency } \\
\hline & & & & & A & B & $\mathrm{C}$ & $\mathrm{AB}$ & $\mathrm{AC}$ & $\mathrm{BC}$ & $\mathrm{ABC}$ \\
\hline \multicolumn{12}{|c|}{ Bacteria } \\
\hline Bacillus subtilis 6051 & 19786 & 0.7 & 0.3 & 656 & 11 & 2 & 0 & 87 & 0 & 0 & 0 \\
\hline Vibrio fischeri 7744 & 15073 & 0.7 & 0.4 & 581 & 97 & 0 & 0 & 1 & 2 & 0 & 0 \\
\hline Bordetella pertussis 9797 & 12834 & 0.5 & 0.4 & 84 & 93 & 0 & 1 & 1 & 3 & 0 & 1 \\
\hline Thiobacillus thiooxidans 19377 & 10100 & 0.9 & 0.4 & 178 & 94 & 0 & 0 & 1 & 3 & 0 & 2 \\
\hline Escherichia coli $\mathrm{K}-12$ & 11900 & 0.9 & 0.4 & 265 & 95 & 0 & 0 & 1 & 3 & 0 & 1 \\
\hline Pseudomonas aeruginosa 10145 & 28190 & 0.4 & 0.1 & 248 & 94 & 0 & 0 & 1 & 3 & 0 & 2 \\
\hline Acinetobacter baumannii 19606 & 30006 & 1.1 & 0.5 & 556 & 96 & 0 & 0 & 0 & 3 & 0 & 1 \\
\hline Micrococcus luteus 4698 & 28197 & 0.9 & 0.4 & 502 & 97 & 0 & 0 & 1 & 2 & 0 & 0 \\
\hline Mycobacterium parafortuitum 19686 & 18197 & 0.4 & 0.2 & 265 & 90 & 0 & 0 & 8 & 0 & 0 & 2 \\
\hline Staphylococcus aureus 12600 & 30015 & 0.7 & 0.2 & 283 & 96 & 0 & 0 & 2 & 1 & 0 & 1 \\
\hline Burkholderia cepacia 25416 & 30003 & 0.5 & 0.2 & 399 & 97 & 0 & 0 & 2 & 1 & 0 & 0 \\
\hline Pseudomonas aeruginosa GFP 15692 & 16015 & 0.6 & 0.3 & 112 & 97 & 0 & 0 & 1 & 1 & 0 & 1 \\
\hline Serratia marcescens (DSMZ 3021) & 30027 & 0.6 & 0.2 & 393 & 96 & 0 & 0 & 3 & 1 & 0 & 0 \\
\hline Enterococcus faecalis 19433 & 17401 & 0.8 & 0.3 & 327 & 96 & 0 & 0 & 1 & 1 & 0 & 2 \\
\hline \multicolumn{12}{|c|}{ Fungi } \\
\hline Aspergillus niger (28 days) 16888 & 30772 & 4.4 & 2 & 157 & 79 & 2 & 7 & 4 & 2 & 2 & 5 \\
\hline Aspergillus niger (180 days) 16888 & 23086 & 4.4 & 2 & 181 & 78 & 2 & 5 & 5 & 2 & 2 & 5 \\
\hline Aspergillus versicolor 9577 & 32620 & 4 & 2.1 & 304 & 67 & 2 & 0 & 9 & 0 & 8 & 14 \\
\hline Aspergillus flavus 16883 & 15847 & 4 & 1.3 & 364 & 87 & 0 & 0 & 3 & 1 & 2 & 4 \\
\hline Aspergillus pinenceus (sp. proposed) & 33085 & 3.6 & 1.1 & 206 & 90 & 0 & 0 & 5 & 0 & 0 & 3 \\
\hline Aspergillus sydowii 16844 & 5098 & 3.5 & 1.4 & 515 & 90 & 0 & 0 & 5 & 0 & 0 & 3 \\
\hline Aspergillus tubingensis 10550 & 28801 & 2.5 & 1.5 & 172 & 41 & 7 & 7 & 25 & 0 & 5 & 12 \\
\hline Botrytis spp. & 7024 & 8 & 3.7 & 294 & 21 & 6 & 0 & 55 & 0 & 0 & 16 \\
\hline Cheatomium spp. & 19264 & 4.9 & 2.1 & 258 & 77 & 0 & 0 & 16 & 0 & 1 & 3 \\
\hline Cladosporium spp. & 39294 & 3.3 & 1 & 710 & 80 & 0 & 0 & 16 & 0 & 0 & 2 \\
\hline Cladosporium cladosporioides (28 days) 16022 & 26474 & 3 & 0.9 & 570 & 72 & 0 & 0 & 24 & 0 & 0 & 3 \\
\hline Cladosporium cladosporioides (180 days) 16022 & 34085 & 3.3 & 1.2 & 702 & 70 & 0 & 0 & 23 & 0 & 0 & 4 \\
\hline Cladosporium herbarum 58927 & 2962 & 3.2 & 1.4 & 651 & 80 & 0 & 0 & 12 & 0 & 0 & 6 \\
\hline Fusarium spp. & 1188 & 3.3 & 1.5 & 638 & 81 & 0 & 0 & 12 & 0 & 0 & 5 \\
\hline Paecilomyces variotii 18502 & 11188 & 4.2 & 1.5 & 772 & 0 & 8 & 0 & 74 & 0 & 3 & 13 \\
\hline Penicillium chrysogenum (28 days) 10106 & 14577 & 3.5 & 1.1 & 867 & 92 & 0 & 0 & 4 & 0 & 0 & 2 \\
\hline Penicillium chrysogenum (180 days) 10106 & 33452 & 3.2 & 1 & 733 & 73 & 0 & 0 & 23 & 0 & 0 & 2 \\
\hline Penicillium canescens 10419 & 7840 & 3.1 & 1.3 & 659 & 90 & 0 & 0 & 5 & 0 & 0 & 4 \\
\hline Penicillium citreonigrum 22350 & 8470 & 2.8 & 1 & 596 & 68 & 0 & 0 & 27 & 0 & 0 & 4 \\
\hline Penicillium commune 10428 & 9255 & 3.3 & 1.3 & 762 & 79 & 0 & 0 & 3 & 2 & 0 & 14 \\
\hline Penicillium corylophilum 9784 & 12654 & 2.6 & 0.9 & 687 & 90 & 0 & 0 & 6 & 0 & 0 & 3 \\
\hline Penicillium cecumbens (sp. proposed) & 13676 & 2.4 & 0.5 & 420 & 89 & 0 & 0 & 8 & 0 & 0 & 2 \\
\hline (50) Penicillium thomii 18313 & 5739 & 3.1 & 0.9 & 721 & 91 & 0 & 0 & 4 & 0 & 0 & 3 \\
\hline Phoma herbarum (28 days) 44739 & 5518 & 2.9 & 1.2 & 607 & 91 & 0 & 0 & 5 & 0 & 0 & 2 \\
\hline Phoma herbarum (180 days) 44739 & 2648 & 3.5 & 1.9 & 688 & 79 & 0 & 0 & 14 & 0 & 0 & 5 \\
\hline Stachybotrys spp. & 12697 & 2.8 & 1.2 & 137 & 65 & 7 & 2 & 14 & 1 & 1 & 6 \\
\hline Syncephalastrum racemosum 14832 & 6753 & 2.8 & 2.3 & 618 & 75 & 0 & 0 & 12 & 0 & 0 & 10 \\
\hline Tritirachium sp. & 5595 & 2.2 & 0.9 & 227 & 95 & 0 & 0 & 3 & 0 & 0 & 0 \\
\hline Ulocladium sp. & 4513 & 3.8 & 1.7 & 767 & 80 & 0 & 0 & 15 & 0 & 0 & 4 \\
\hline \multicolumn{12}{|c|}{ Pollens } \\
\hline Acer saccharum & 800 & 3.3 & 4.9 & 753 & 6 & 1 & 3 & 1 & 1 & 17 & 68 \\
\hline Alnus rubra and rugosa mix & 389 & 4.7 & 3.1 & 387 & 6 & 4 & 11 & 1 & 0 & 28 & 46 \\
\hline Ambrosia trifida & 1219 & 3.2 & 4.7 & 876 & 11 & 2 & 2 & 6 & 1 & 12 & 62 \\
\hline Artemisia tridentata & 1703 & 2.5 & 4.1 & 666 & 6 & 2 & 2 & 3 & 0 & 19 & 66 \\
\hline Betula lenta, nigra \& populifolia & 2208 & 2.5 & 4.2 & 990 & 4 & 1 & 3 & 2 & 0 & 54 & 33 \\
\hline Carya laciniosa & 1942 & 1.3 & 2.1 & 1043 & 5 & 0 & 5 & 2 & 0 & 53 & 33 \\
\hline Eucalyptus & 1095 & 6.1 & 5 & 481 & 2 & 1 & 13 & 0 & 0 & 47 & 34 \\
\hline Fern (unknown source) & 1571 & 6.8 & 5.7 & 373 & 1 & 13 & 17 & 1 & 0 & 38 & 28 \\
\hline Fagus americana & 1991 & 1.8 & 2.4 & 1155 & 6 & 2 & 6 & 3 & 0 & 60 & 20 \\
\hline Juglans nigra & 1968 & 1.2 & 2.2 & 1197 & 3 & 1 & 4 & 1 & 0 & 62 & 26 \\
\hline Juniperus ashei & 2536 & 3 & 5.2 & 766 & 0 & 1 & 1 & 0 & 0 & 16 & 79 \\
\hline Morus rubra & 4232 & 2.8 & 5.5 & 1229 & 0 & 0 & 0 & 0 & 0 & 1 & 96 \\
\hline Phleum pratense & 3390 & 1.5 & 3.4 & 936 & 2 & 0 & 1 & 0 & 0 & 11 & 82 \\
\hline
\end{tabular}


Acknowledgements. This work is the result of a collaboration of senior instrument developers, agency researchers and academics in the form of an interagency, university-industry cooperative. It was funded in part by a grant from the National Science Foundation, Division of Bioenvironmental Sciences (BES 1134594), and by in-kind contributions from Droplet Measurement Technologies, Research and Development group. Salary support for A. Perring was from the NOAA Atmospheric Composition and Climate Program and the NOAA Health of the Atmosphere Program.

Edited by: F. Pope

Reviewed by: J. Huffman and one anonymous referee

\section{References}

Agranovski, V., Ristovski, Z., Hargreaves, M., J. Blackall, P., and Morawska, L.: Performance evaluation of the UVAPS: influence of physiological age of airborne bacteria and bacterial stress, J. Aerosol Sci., 34, 1711-1727, 2003.

Bovallius, A., Bucht, B., Roffey, R., and Anas, P.: Long-range air transmission of bacteria, Appl. Environ. Microbiol., 35, 12311232, 1978.

Burrows, S. M., Elbert, W., Lawrence, M. G., and Pöschl, U.: Bacteria in the global atmosphere - Part 1: Review and synthesis of literature data for different ecosystems, Atmos. Chem. Phys., 9, 9263-9280, doi:10.5194/acp-9-9263-2009, 2009.

Crawford, I., Ruske, S., Topping, D. O., and Gallagher, M. W.: Evaluation of hierarchical agglomerative cluster analysis methods for discrimination of primary biological aerosol, Atmos. Meas. Tech., 8, 4979-4991, doi:10.5194/amt-8-4979-2015, 2015.

D'Amato, G., Liccardi, G., and Frenguelli, G.: Thunderstormasthma and pollen allergy, Allergy, 62, 11-16, 2007.

Despres, V. R., Huffman, J. A., Burrows, S. M., Hoose, C., Safatov, A. S., Buryak, G., Froehlich-Nowoisky, J., Elbert, W., Andreae, M. O., Poeschl, U., and Jaenicke, R.: Primary biological aerosol particles in the atmosphere: a review, Tellus B, 64, doi:10.3402/tellusb.v64i0.15598, 2012.

Gabey, A. M., Gallagher, M. W., Whitehead, J., Dorsey, J. R., Kaye, P. H., and Stanley, W. R.: Measurements and comparison of primary biological aerosol above and below a tropical forest canopy using a dual channel fluorescence spectrometer, Atmos. Chem. Phys., 10, 4453-4466, doi:10.5194/acp-10-4453-2010, 2010.

Gabey, A. M., Vaitilingom, M., Freney, E., Boulon, J., Sellegri, K., Gallagher, M. W., Crawford, I. P., Robinson, N. H., Stanley, W. R., and Kaye, P. H.: Observations of fluorescent and biological aerosol at a high-altitude site in central France, Atmos. Chem. Phys., 13, 7415-7428, doi:10.5194/acp-13-7415-2013, 2013.

Heald, C. L. and Spracklen, D. V.: Atmospheric budget of primary biological aerosol particles from fungal spores, Geophys. Res. Lett., 36, L09806, doi:10.1029/2009gl037493, 2009.

Healy, D. A., O'Connor, D. J., Burke, A. M., and Sodeau, J. R.: A laboratory assessment of the Waveband Integrated Bioaerosol Sensor (WIBS-4) using individual samples of pollen and fungal spore material, Atmos. Environ., 60, 534-543, 2012.

Hernandez, M., Miller, S. L., Landfear, D., and Macher, J.: A combined fluorochrome method for quantitation of metabolically active and inactive airborne bacteria, Aerosol Sci. Technol., 30, 145-160, 1999 .
Hill, T. C. J., Moffett, B. F., DeMott, P. J., Georgakopoulos, D. G., Stump, W. L., and Franc, G. D.: Measurement of Ice NucleationActive Bacteria on Plants and in Precipitation by Quantitative PCR, Appl. Environ. Microbiol., 80, 1256-1267, 2014.

Huffman, J. A., Treutlein, B., and Pöschl, U.: Fluorescent biological aerosol particle concentrations and size distributions measured with an Ultraviolet Aerodynamic Particle Sizer (UVAPS) in Central Europe, Atmos. Chem. Phys., 10, 3215-3233, doi:10.5194/acp-10-3215-2010, 2010.

Hurst, C. J., Crawford, R. L., Garland, J. L., and Lipson, D. A.: Manual of environmental microbiology, American Society for Microbiology Press, 2007.

Kanaani, H., Hargreaves, M., Ristovski, Z., and Morawska, L.: Performance assessment of UVAPS: Influence of fungal spore age and air exposure, J. Aerosol Sci., 38, 83-96, 2007.

Kaye, P. H., Stanley, W. R., Hirst, E., Foot, E. V., Baxter, K. L., and Barrington, S. J.: Single particle multichannel bio-aerosol fluorescence sensor, Optics Express, 13, 3583-3593, 2005.

Kepner, R. L. and Pratt, J. R.: Use of fluorochromes for direct enumeration of total bacteria in environmental samples: past and present, Microbiol. Rev., 58, 603-615, 1994.

Lakowicz, E. R.: Principles of Fluorescence Spectroscopy, Springer Science and Buisness Media, Berlin, 2006.

Lighthart, B.: The ecology of bacteria in the alfresco atmosphere, FEMS Microbiol. Ecol., 23, 263-274, 1997.

Madigan, M. T., Clark, D. P., and Martinko, J. M.: Brock Biology of Microorganisms. New York, New York, USA, 2011.

Madigan, M. T., Martinko, J. M., Dunlap, P. V., and Clark, D. P.: Brock Biology of microorganisms 12th Edn., Int. Microbiol., 11, 65-73, 2008

Miguel, A. G., Taylor, P. E., House, J., Glovsky, M. M., and Flagan, R. C.: Meteorological influences on respirable fragment release from Chinese elm pollen, Aerosol Sci. Technol., 40, 690-696, 2006.

O'Connor, D. J., Healy, D. A., Hellebust, S., Buters, J. T. M., and Sodeau, J. R.: Using the WIBS-4 (Waveband Integrated Bioaerosol Sensor) technique for the on-line detection of pollen grains, Aerosol Sci. Technol., 48, 341-349, 2013.

Pan, Y.-L.: Detection and characterization of biological and other organic-carbon aerosol particles in atmosphere using fluorescence, J. Quant. Spectros. Radiat. Transfer, 150, 12-35, 2015.

Pan, Y.-L., Hill, S. C., Pinnick, R. G., Huang, H., Bottiger, J. R., and Chang, R. K.: Fluorescence spectra of atmospheric aerosol particles measured using one or two excitation wavelengths: Comparison of classification schemes employing different emission and scattering results, Optics Express, 18, 12436-12457, 2010.

Peccia, J., H., W., Miller, S., and Hernandez, M.: Effects of relative humidity on the ultraviolet induced inactivation of airborne bacteria, Aersol Sci. Technol., 70, 969-990, 2000.

Peccia, J. and Hernandez, M.: Incorporating polymerase chain reaction-based identification, population characterization, and quantification of microorganisms into aerosol science: a review, Atmos. Environ., 40, 3941-3961, 2006.

Perring, A. E., Schwarz, J. P., Baumgardner, D., Hernandez, M. T., Spracklen, D. V., Heald, C. L., Gao, R. S., Kok, G., McMeeking, G. R., McQuaid, J. B., and Fahey, D. W.: Airborne observations of regional variation in fluorescent aerosol across the United States, J. Geophys. Res.-Atmos., 120, 1153-1170, 2015. 
Pinnick, R., Fernandez, E., Rosen, J., Hill, S., Wang, Y., and Pan, Y.: Fluorescence spectra and elastic scattering characteristics of atmospheric aerosol in Las Cruces, New Mexico, USA: Variability of concentrations and possible constituents and sources of particles in various spectral clusters, Atmos. Environ., 65, 195-204, 2013.

Pöhlker, C., Huffman, J. A., and Pöschl, U.: Autofluorescence of atmospheric bioaerosols - fluorescent biomolecules and potential interferences, Atmos. Meas. Tech., 5, 37-71, doi:10.5194/amt-537-2012, 2012.

Poschl, U., Martin, S. T., Sinha, B., Chen, Q., Gunthe, S. S., Huffman, J. A., Borrmann, S., Farmer, D. K., Garland, R. M., Helas, G., Jimenez, J. L., King, S. M., Manzi, A., Mikhailov, E., Pauliquevis, T., Petters, M. D., Prenni, A. J., Roldin, P., Rose, D., Schneider, J., Su, H., Zorn, S. R., Artaxo, P., and Andreae, M. O.: Rainforest Aerosols as Biogenic Nuclei of Clouds and Precipitation in the Amazon, Science, 329, 1513-1516, 2010.

Saari, S., Mensah-Attipoe, J., Reponen, T., Veijalainen, A. M., Salmela, A., Pasanen, P., and Keskinen, J.: Effects of fungal species, cultivation time, growth substrate, and air exposure velocity on the fluorescence properties of airborne fungal spores, Indoor Air, 25, 653-661, doi:10.1111/ina.12166, 2014.

Shaffer, B. T. and Lighthart, B.: Survey of culturable airborne bacteria at four diverse locations in Oregon: Urban, rural, forest, and coastal, Microb. Ecol., 34, 167-177, 1997.

Sivaprakasam, V., Lin, H.-B., Huston, A. L., and Eversole, J. D.: Spectral characterization of biological aerosol particles using two-wavelength excited laser-induced fluorescence and elastic scattering measurements, Optics Express, 19, 6191-6208, 2011.

Sivasubramani, S., K., Niemeier, R., T., Reponen, T., and Grinshpun, S., A.: Fungal spore source strength tester: laboratory evaluation of a new concept, Sci. Total Environ., 329, 75-86, 2004.

Stone, R. C. and Johnson, D. L.: A Note on the Effect of Nebulization Time and Pressure on the Culturability of Bacillus subtilis and Pseudomonas fluorescens, Aerosol Sci. Technol., 36, 536539, 2002.

Taylor, P. E., Flagan, R. C., Miguel, A. G., Valenta, R., and Glovsky, M. M.: Birch pollen rupture and the release of aerosols of respirable allergens, Clinical \& Experimental Allergy, 34, 15911596, 2004.
Taylor, P. E., Flagan, R. C., Valenta, R., and Glovsky, M. M.: Release of allergens as respirable aerosols: A link between grass pollen and asthma, Journal of Allergy and Clinical Immunology, 109, 51-56, 2002.

Taylor, P. E., Jacobson, K. W., House, J. M., and Glovsky, M. M.: Links between pollen, atopy and the asthma epidemic, International Archives of Allergy and Immunology, 144, 162-170, 2007.

Thomas, R. J., Webber, D., Hopkins, R., Frost, A., Laws, T., Jayasekera, P. N., and Atkins, T.: The cell membrane as a major site of damage during aerosolization of Escherichia coli, Appl. Environ. Microbiol., 77, 920-925, 2011.

Tong, Y. Y. and Lighthart, B.: The annual bacterial particle concentration and size distribution in the ambient atmosphere in a rural area of the Willamette valley, Oregon, Aerosol Sci. Technol., 32, 393-403, 2000.

Toprak, E. and Schnaiter, M.: Fluorescent biological aerosol particles measured with the Waveband Integrated Bioaerosol Sensor WIBS-4: laboratory tests combined with a one year field study, Atmos. Chem. Phys., 13, 225-243, doi:10.5194/acp-13225-2013, 2013.

Uk Lee, B., Jung, J. H., Yun, S. H., Hwang, G. B., and Bae, G. N.: Application of UVAPS to real-time detection of inactivation of fungal bioaerosols due to thermal energy, J. Aerosol Sci., 41, 694-701, 2010.

Vesper, S., McKinstry, C., Haugland, R., Wymer, L., Bradham, K., Ashley, P., Cox, D., Dewalt, G., and Friedman, W.: Development of an Environmental Relative Moldiness index for US homes, Journal of Occupational and Environmental Medicine, 49, 829833, 2007.

Wang, C.-C., Fang, G.-C., and Lee, L.-Y.: The study of ambient air bioaerosols during summer daytime and nighttime periods in Taichung, Central Taiwan, Environmental Forensics, 9, 6-14, 2008. 